\title{
Hollywood, Atome et Guerre froide. Entre détente et terreur
}

\author{
Hollywood, Atom and Cold War. Between Detente and Terror
}

\author{
Robert Nardone ${ }^{1}$ \\ ${ }^{1}$ Laboratoire Histoire des TechnoSciences en Société, Conservatoire national des arts et métiers, \\ robert.nardone@lecnam.net
}

RÉSUMÉ. Notre article se propose d'examiner trois temps distincts de la Guerre froide à l'aune de films hollywoodiens apocalyptiques produits et diffusés dans ces trois mêmes périodes. De quoi sont concrètement faits les liens intimes et directs entre un conflit politique, idéologique et militaire et sa représentation cinématographique immédiate ? Comment le script, le choix du format (N\&B ou couleur), le choix du décor, des costumes, de la bande son, des acteurs et de la mise en scène, nous renseignent-ils sur l'état précis, à un instant donné, des relations belligérantes entre deux Etats ? En quoi ces éléments qui relèvent de l'esthétique nous renseignent-ils sur les positions politiques des réalisateurs, quand la censure est à son plus haut degré d'intensité ?

ABSTRACT. Our article proposes to examine three distinct Cold War periods in the light of apocalyptic Hollywood films produced and broadcast in the same three periods. What are the concrete intimate and direct links between a political, ideological and military conflict and its immediate cinematographic representation? How do the script, the choice of format (B\&W or color), the choice of the decor, the costumes, the soundtrack, the actors and the staging, inform us about the precise state, at a given moment, of the belligerent relations between two states? How do these aesthetic elements inform us about the political positions of directors, when censorship is at its highest intensity? MOTS-CLÉS. Hollywood, Bombe atomique, Guerre froide, Destruction mutuelle assurée, propagande, géopolitique, angoisse, détente, suspense.

KEYWORDS. Hollywood, Atomic Bomb, Cold War, Mutual Assured Destruction, Propaganda, Geopolitics, Anxieti, Detente, Suspense.

\section{In memoriam Marc Ferro}

« Les effets furent sans précédents, beaux, magnifiques, stupéfiants et terrifiants... Une lumière dévorante, un grondement persistant et lugubre, pareil à un avertissement du Jugement dernier. À ce tonnerre, nous comprîmes que nous avions eu, êtres infimes, l'audace sacrilège de toucher aux forces jusque-là réservées au Tout-Puissant. » ${ }^{1}$

Général Thomas Farrell.

Tout au long de la Guerre froide, les Etats-Unis et l'URSS ont fait vivre le monde dans l'inquiétude d'une destruction nucléaire totale et irréversible (Soutou, 2001 ; Gaddis, 2005 ; Westad, 2017). ${ }^{2}$ Ils l'ont fait à travers des déclarations et des pourparlers médiatisés par la presse, la radio et la télévision, mais aussi par une importante production cinématographique (Puiseux, 1988) ${ }^{3}$. Le

\footnotetext{
${ }^{1}$ Général Thomas Farrell, cité par Robert Jungk, Brighter than a Thousand Suns: A Personal History of the Atomic Scientists, 1958.

${ }^{2}$ Soutou, G.-H., La Guerre de Cinquante Ans, Les relations Est-Ouest, 1943-1990, Fayard, 2001. Gaddis, J.L., The Cold War: A New History, The Penguin Press, 2005. Westad, O.A. The Cold War: A World History, Allen Lane, 2017.

${ }^{3}$ Puiseux, H., L'apocalypse nucléaire et son cinéma, Cerf, col. 7e Art, 1988. L'auteur a identifié une centaine de films sur ce thème. Elle fait remarquer qu'aucun de ces films n'a été produit par les Soviétiques trop occupés, écrit-elle, à faire la propagande de la supériorité sociale du régime.
} 
cinéma hollywoodien, habitué à transformer en fiction chaque événement d'importance (Vernier, $2003)^{4}$, s'empare très tôt ${ }^{5}$ de cette situation eschatologique si cinégénique. La fin du monde dont il s'agit ici renvoie à une menace bien réelle ${ }^{6}$. Elle est fondée sur une stratégie militaire, idéologique et politique, celle dite de l'Equilibre de la terreur ou Destruction mutuelle assurée (Allison, 1971; Follen et $a l, 1997)^{7}$. Et ce spectre apocalyptique fait son cinéma (Puiseux, 1988). L'ombre d'une explosion atomique en chaîne plane alors sur le monde, entrainant un sentiment d'insécurité quasipermanent. Ce cataclysme est possible et imminent, mais où et quand cela adviendra-t-il ? Une aubaine pour le cinéma qui sait faire de ces deux inconnues - où et quand - un moteur narratif : le suspense (Bessalel, Gardies, 1994 ; Hitchcock, 1983) ${ }^{8}$. «Maintenant, examinons le suspense. La bombe est sous la table et le public le sait. Le public sait que la bombe explosera à une heure et il sait qu'il est une heure moins le quart - il y a une horloge dans le décor - une même conversation anodine devient tout à coup très intéressante parce que le public participe à la scène (...). Dans ce cas, nous lui offrons quinze minutes de suspense. $»^{9}$ (Hitchcock, 1983).

Ce suspense crée ici une angoisse qui ne concerne pas que les personnages du film, ni même le seul spectateur pris par l'action, mais l'humanité tout entière. Il provoque dès lors, chez ce spectateur, une anxiété élevée au carré, substituant ainsi l'émotion à la raison ${ }^{10}$. Comme si le récit mis en œuvre dans ces films jouait un double rôle, celui de vecteur de propagande, en même temps que celui d'une possible catharsis. Quels que soient les prédicats utilisés pour décrire les divers moments de cette période historique, les termes choisis introduisent une dimension propre au récit fictionnel hollywoodien classique, trois moments qui le structurent : la menace - ici sous la forme de la terreur, ou de la destruction assurée - ; le suspense - ici sous la forme d'équilibre des forces, ou de destruction mutuelle - et le climax (Baroni, 2016; Cayatte, Goudman, 2020) ${ }^{11}$. Si l'imminence d'une eschatologie d'origine nucléaire réside dans le combat des forces du Bien incarnées par ce que je qualifierais de libéralisme religieux de l'administration américaine ${ }^{12}$, contre

\footnotetext{
${ }^{4}$ Vernier J.-M., Cinéma et Amérique : une image effritée. In: Quaderni, n50-51, Printemps 2003. Images de l'Amérique du Nord vues par elle-même ou vues par les autres pp. 197-209. DOI :

https://doi.org/10.3406/quad.2003.1228,www.persee.fr/doc/quad_0987-1381_2003_num_50_1_1228

${ }^{5}$ Dès 1959 avec On the Beach et avec la crise des missiles de Cuba (1962) : Dr Strange love et Fail safe (1964).

${ }^{6}$ Parmi les régulières prédictions de fin du monde annoncées par des illuminés, on ne compte pas moins de cinq prédictions de fin du monde provoquée par une catastrophe nucléaire entre 1967 et 1980.
}

${ }^{7}$ Fohlen, C., Heffer, J. \& Weil, F. (1997). De la diplomatie aux relations internationales. Dans : C. Fohlen, J. Heffer \& F. Weil (Dir), Canada et États-Unis depuis 1770 (pp. 413-448). Paris cedex 14, France: Presses Universitaires de France. Graham T. Allison, Essence of Decision: Explaining the Cuban Missile Crisis, Little Brown, 1971.

${ }^{8}$ Bessalel, J., Gardies, A., (dir.), Le suspense au cinéma, CinémAction, nº 71, Corlet Publications, 1994,

${ }^{9}$ Truffaut, F., Hitchcock/Truffaut, Ramsay, 1983, pp.57-58. Pour Hitchcock : "les émotions sont un ingrédient nécessaire au suspense ».

${ }^{10}$ Le nombre d'abris antiatomiques qui se compte encore, dans le monde, par centaines de milliers, publics ou privés, donne bien la mesure de la peur engendrée par l'affrontement nucléaire potentiel entre les deux puissances.

11 Baroni, R., Pour une narratologie transmédiale, https://serval.unil.ch/resource/serval:BIB_C51203E3B809.P001/REF.pdf. Cayatte, R., et Goudmand, A., « Approches transmédiales du récit dans les fictions contemporaines », Cahiers de Narratologie [En ligne], 37 | 2020, mis en ligne le 04 septembre 2020, URL : http://journals.openedition.org/narratologie/10392 ; DOI : https://doi.org/10.4000/narratologie.10392

${ }^{12}$ Le premier amendement prévoit que "Le Congrès ne pourra faire aucune loi concernant l'établissement d'une religion (clause d'établissement) ou interdisant son libre exercice (clause de libre exercice)." Mais il s'agit bien d'une « laïcité philo-cléricale » pour reprendre la juste expression de Denis Lacorne, « Une laïcité à l'américaine », Études, 2008/10 (Tome 409), p. 297-305. DOI : 10.3917/etu.094.0297. URL : https://www.cairn.info/revue-etudes-2008-10-page-297.htm. 
celles de l'Empire du Mal (Reagan, 1983) ${ }^{13}$ incarné par le communisme mécréant des soviets, la production cinématographique ne s'avère pas aussi tranchée et caricaturale dans ses positions politiques.

Ces productions américaines mettant en scène des moments précis de la Guerre froide, entrainent des changements de point de vue selon l'évolution historique, les rapports de force et l'état des relations diplomatiques. Ces variations ont le mérite de nuancer les injonctions gouvernementales, venant de l'administration Truman et ses successeurs tout au long de la période étudiée ${ }^{14}$, à faire des médias en général et du cinéma en particulier un vecteur de propagande anti-communiste (Lepri, 2010) ${ }^{15}$. Les scénaristes et les cinéastes se trouvent-ils alors seulement dans une situation historique qui leur offre les ingrédients proprement narratifs, conformes aux canons binaires hollywoodiens (Baroni, 2016), comme les westerns, les films policiers ou les films de science-fiction en ont tant produits ? S'y retrouve ces deux protagonistes archétypaux que tout oppose, l'un incarnant : le bon, le droit, la vérité, la liberté, la justice ; l'autre portant fatalement en lui : le mal, la fourberie, le mensonge, la dictature, l'injustice, sur fond de référence biblique dans un Armageddon à l'échelle planétaire. Ou bien se trouvent-ils aussi dans une situation qui les contraigne, à des degrés divers et selon les variations des relations, à intégrer dans leurs films tous les éléments qui construisent un discours propagandiste mais en le recombinant à leurs façons artistiques et critiques ? Peut-être alors verrons-nous apparaître un balancement, entre ce discours-là et un autre discours qui, parce qu'il emprunte la voie artistique propre au cinéma, serait plus géopolitique que propagandiste ? Autrement dit, à travers la seule étude artistique de ces films, est-il possible d'évaluer les degrés de terreur et de détente, et de mesurer leur degré de propagande tels qu'ils ont été appréhendés par des cinéastes, témoignant ainsi de l'état d'une partie de la production américaine ? Sans perdre de vue qu'Hollywood est encore sous haute surveillance policière, malgré la fin de la période maccarthyste.

Dans un premier temps j'exposerai mon corpus et j'en justifierai les choix. Dans un second temps, je contextualiserai le conflit et l'usage qui a été fait ou non de ce genre cinématographique comme outil de propagande. Enfin, avec l'analyse même des films, je montrerai comment ils peuvent à eux seuls rendre compte avec précision des divers états du conflit historique.

\section{Le cinéma comme agent de l'histoire}

En me fondant sur l'idée selon laquelle le cinéma peut jouer ce rôle-là d'agent de l'histoire (Ferro, 1977) ${ }^{16}$, je vais me concentrer sur l'analyse de films (Bellour, 1995 ; Odin, 2000) ${ }^{17}$ qui ont mis en scène trois moments précis du conflit entre 1949 et 1989. Pour Antoine de Baecque «Le cinéma semble être devenu... la forme moderne de la représentation historique ${ }^{18}$. Mon corpus est

\footnotetext{
13 «Evil empire ». «L'empire du mal», Ronald Reagan, 1983. «Dans vos discussions sur les propositions de gel nucléaire, je vous exhorte à prendre garde à la tentation de l'orgueil, à la tentation de vous déclarer allègrement au-dessus de tout cela et à blâmer les deux parties également, à ignorer les faits de l'histoire et les impulsions agressives d'un empire mauvais, pour simplement appeler la course aux armements un énorme malentendu et ainsi vous retirer de la lutte entre ce qui est le juste et l'injuste et le bien et le mal."
}

14 « Nous devons nous faire entendre dans le monde entier dans une grande campagne de vérité. » Le président des Etats-Unis, Harry S. Truman, Letter to Senator Flanders on the Appropriation for the Campaign of Truth, 1950.

\footnotetext{
${ }^{15}$ Lepri, C., « De l'usage des médias à des fins de propagande pendant la guerre froide », Revue internationale et stratégique, 2010/2 (n ${ }^{\circ}$ 78), p. 111-118. DOI : 10.3917/ris.078.0111. URL : https://www.cairn.info/revue-internationale-et-strategique-2010-2page-111.htm
}

${ }^{16}$ C'est Marc Ferro qui propose cette élégante formule dans Cinéma et Histoire, Denoël et Gonthier, 1977.

\footnotetext{
${ }^{17}$ Bellour, R., L’Analyse du film, Calmann-Lévy, 1995. Odin, R., De la fiction, De Boeck Université, 2000.

${ }^{18}$ Ferro, M., Analyse de film, analyse de sociétés : une source nouvelle pour l'Histoire, Hachette, 1974 ; Cinéma et Histoire, op.cit., 1977 ; Film et histoire, Éditions de l'EHESS, 1984. Metz, C., Essais sur la signification au cinéma, Klincksieck, 1973. Bellour, R., L'Analyse du film, Calmann-Lévy, 1979, 1995. Aumont, J., (avec Michel Marie) L'Analyse des films, Nathan, 1988. De Baecque, A. L'Histoire-caméra, Gallimard, 2008.
} 
constitué de six films qui ont été produits et diffusés massivement dans la sphère mondiale qui était alors sous influence américaine, pendant toute la période de la Guerre froide. Ils ont en commun d'utiliser l'arme atomique comme l'élément moteur d'un anéantissement général (Parmentier, 1993 ; Tertraits, 2008) ${ }^{19}$.

Je propose d'analyser ce corpus en couplant les films : On the Beach (1959) de Stanley Kramer et The Day after (1983) de Nicholas Meyer; Dr. Strangelove (1964) de Stanley Kubrick et Fail Safe (1964) de Sidney Lumet; You Only Live Twice (1967) et The Spy Who Loved Me (1977) de Lewis Gilbert.

Parmi l'ensemble des films-catastrophe, films d'espionnage et films de science-fiction mettant en scène la bombe atomique, deux particularités caractérisent et justifient mes choix :

- La première, tient dans la facture même des films, dans leur esthétique, dans leur popularité ${ }^{20}$, dans leur simultanéité avec les événements historiques, mais aussi parce qu'il était possible de les faire fonctionner en binôme. Chaque film du couple correspond à un même état des relations entre les belligérants et par des éléments dramaturgiques communs. On the Beach et The Day after, bien que séparés par deux décennies, sortent dans un même type de tension mondiale. Dans l'un comme dans l'autre, la catastrophe est en train d'avoir lieu et c'est la population face à l'apocalypse qui en est l'acteur central, quand elle est totalement absente des quatre autres films. Dr. Strangelove et Fail Safe, paraissent tout de suite après deux incidents qui ont bien failli déclencher l'apocalypse. Dans l'un comme dans l'autre, ce sont les autorités, les cas de conscience, les prises de décision au plus haut niveau de l'Etat face à un choix politico-militaire tragique qui concentrent toute l'attention. Les deux James Bond, paraissent l'un en pleine Détente, tandis que l'autre annonce sa fin. Ils mettent en scène une relation entre deux personnages dépsychologisés $\left(\right.$ Flemming, 1953 ; Eco, 1966) ${ }^{21}$, qui se retrouvent à combattre un tiers méchant voulant dominer le monde avec la menace atomique.

- La deuxième particularité tient dans la simultanéité entre l'événement et sa représentation cinématographique $^{22}$. Autrement dit ces films rendent compte spontanément de l'état des relations politico-militaires entre les deux Etats à un moment donné. Les historiens distinguent trois périodes, caractérisées par des changements importants dans les relations diplomatiques et militaires entre les Etats-Unis et l'URSS : la Coexistence pacifique (1956-1962), la Détente (1963$1974 / 1979)$ et la Seconde Guerre froide $(1975 / 1979-1984)^{23}$. Ce qui a conduit à un balancement

\footnotetext{
${ }^{19}$ Parmentier G., Le retour de l'Histoire. Stratégie et relations internationales pendant et après la Guerre froide. Éditions Complexe (programme ReLIRE), "Questions au XXe siècle », 1993, 306 pages. ISBN : 9782870274835. DOI : 10.3917/comp.parme.1993.01. URL: https://www.cairn.info/le-retour-de-1-histoire--9782870274835.htm. Tertrais, B., L'arme nucléaire. Presses Universitaires de France, 2008.

${ }^{20}$ Les films Dr. Strangelove (Entrées France, 768 573) et On the Beach, ont été couverts de prix, Fail Safe très bien accueilli par la critique a souffert de la sortie la même année du Kubrick. You Only Live Twice et The Spy Who Loved Me, comptent les spectateurs par dizaine de millions. (Entrées monde, 111600000 pour l'un et 185400000 pour l'autre. Chiffres du 6 mai 2007 , source Commanderbond.net).

${ }^{21}$ Fleming, I., Casino Royal, 1953, cité par Eco, U., op.cit., 1966.

${ }^{22}$ La temporalité ici tient compte de l'écart entre l'événement, la réactivité des autorités politiques et la date de sortie des films.

${ }^{23} \mathrm{Si}$ les historiens ne sont pas toujours d'accord sur une date inaugurale de la Guerre froide, ils le sont tous sur sa fin et ils sont d'accord pour identifier cinq périodes dont trois au cours desquelles la présence d'un arsenal atomique important, vectorisé par un déploiement de missiles des deux côtés, va changer le rapport de force et la configuration du conflit. Les périodes qui ne rentrent pas dans notre corpus sont : celle qui voit la constitution des deux parties hétérogènes 1945-1955 et « La Nouvelle détente (19851989) » qui fait suite à la mise en place de La Perestroïka. Cette dernière période du cinéma hollywoodien par temps de Guerre froide mériterait d'être étudiée de manière approfondie. En effet, comme si la victoire des «forces du Bien » était désormais assurée, il apparait un changement d'ennemi dans la mise en scène de l'eschatologie, à cheval sur le thriller catastrophe et la SF. L’ennemi devient métaphorique, il vient du cosmos, des éléments naturels ou du passé.
} 
entre détente et durcissement qui s'est traduit par un climat ambiant et des discours diplomatiques ou propagandistes spécifiques des uns et des autres. Ces trois temps du conflit apparaissent très nettement dans les scénarios et les partis pris de mise en scène de ce corpus. Il s'agit, ici, comme le ferait un spectateur lambda, de s'en tenir au récit fictionnel tel qu'il est dans sa matérialité artistique. Ma proposition a donc pour objet d'utiliser ces productions cinématographiques comme sources archivistiques pertinentes (Ferro, 1977).

\section{Récit historique et récit fictionnel, une rencontre du troisième type}

Il ne me paraît pas absurde d'envisager l'hypothèse que le concept même de " destruction mutuelle assurée » ne pouvait être opérant que par la mise en scène de la terreur par le cinéma et la télévision $^{24}$. Afin de mesurer à quel point se croisent et se confondent, in illo tempore, le récit historique et le récit fictionnel, il convient de rappeler brièvement de quoi est fait le récit historique. Les études historiques sur le sujet se sont confrontées à une double source : celle des archives gouvernementales qui se réfèrent, en l'occurrence, aux négociations et prises de décision des divers acteurs, et celle des archives des organismes de contrôle comme les commissions Rockefeller, Church ou Pike $(1975)^{25}$ qui elles, mettent en évidence le rôle de la propagande. S'appuyant sur une solide littérature américaine, Charlotte Lepri peut avancer que «le recours à la propagande pendant la Guerre froide n'est plus à prouver. Les Etats-Unis et l'Union soviétique l'ont tous deux intensivement utilisée durant cette période, principalement par le biais des médias de masse (cinéma, télévision et radio), dans le but d'étendre au maximum leur zone d'influence. Qui dit Guerre froide pense course aux armements, rideau de fer, dissuasion nucléaire. Mais la lutte que se menèrent les deux blocs fut surtout idéologique et psychologique : la Guerre froide était aussi et peut-être avant tout une guerre d'images, d'idées, de propagande, de désinformation et de pression diplomatique $»^{26}$. Je partage en partie ce point de vue. Pour cadrer l'analyse de mon corpus, il importe de s'y arrêter un instant, car la chose ne va pas de soi. Elle ne va pas de soi d'abord parce que la notion même de propagande, largement étudiée, est un ensemble d'objets plus complexes qu'il n'y paraît. Disons pour faire court et simple, que si l'intentionnalité et les moyens sont nécessaires, ils ne sont pas suffisants. La production du discours, qu'il soit direct ou sous-jacent, passe toujours par des acteurs qui ne sont pas tous au même niveau d'implication et d'un public qui est loin d'être homogène ${ }^{27}$. Enfin, et surtout ici, parce qu'elle est médiatisée par un dispositif hybride qui tout en «produisant autant d'émotion que d'intellection » (Mitry, 2001) ${ }^{28}$ met en œuvre des financements importants qu'il importe de rentabiliser, des structures de production qui doivent durer quel que soit le pouvoir politique, des métiers divers, des hommes et des femmes qui, tout en œuvrant ensemble pour produire des films, ne partagent pas forcément les mêmes opinions. Si Lepri a raison de dire que le recours à la propagande est un fait avéré, il n'en reste pas moins que malgré une forte censure et les retombées, au cours des années soixante, de la très maccarthyste Blacklist,

\footnotetext{
${ }^{24}$ Cette hypothèse se fonde sur notre étude de la médiatisation par le J.T de la « Course à l'espace » entre 1954 et 1984. Quand J.F. Kennedy lance le programme Apollo, il s'assure que les satellites de télécommunication seront opérationnels pour que la Terre entière puisse voir les premiers pas sur la Lune. «Pas de pas sur la Lune sans la télévision ? Cf notre thèse, Le récit de la " Conquête spatiale » par le journal télévisé en temps de Guerre froide (1954-1984) : entre émerveillement \& effroi, 2020.

${ }^{25}$ United States President's Commission on CIA activities within the United States.

${ }^{26}$ Lepri, C., De l'usage des médias à des fins de propagande pendant la guerre froide, Revue internationale et stratégique, $2010 / 2$ $\left(\mathrm{n}^{\circ}\right.$ 78), p. 111-118. DOI : 10.3917/ris.078.0111. URL : https://www.cairn.info/revue-internationale-et-strategique-2010-2-page111.htm

${ }^{27}$ Crozier, M., Friedberg, E., L'acteur et le système, Editions du Seuil, 1977. Dewey, J., Le public et ses problèmes, Editions «Tractacus \& Co », 2005. Nardone, R., Le récit de la conquête spatiale par le journal télévisé en temps de Guerre froide (1954-1984), Thèse, 2020. https://tel.archives-ouvertes.fr/tel-02496108/document

${ }^{28}$ Mitry, J., Esthétique et psychologie du cinéma, les Editions du Cerf, 2001.
} 
certains réalisateurs semblent avoir trouvé, au fil du temps, grâce à la spécificité de l'écriture cinématographique, des moyens pour s'en affranchir ou la détourner.

Entre le 16 et le 28 octobre 1962, les autorités américaines et soviétiques ont dû régler un conflit jusque-là inédit dans l'histoire de l'humanité : le risque d'une attaque nucléaire conjointe. "Le samedi 27 octobre, fut la journée la plus terrifiante qu'aucune autre dont on put se souvenir ${ }^{29}$. Cette frayeur partagée par les acteurs le fut, a posteriori, par le public, essentiellement médiatisée par les films et les actualités télévisées. Le concept même de Mutual Assured Destruction (MAD) par exemple, était surtout connu des autorités militaires, des instances politiques et des chercheurs spécialistes de la Théorie des jeux comme Donald Brennan, Oskar Morgenstern et John von Neumann dont nous verrons qu'il inspira Kubrick. Il n'est pas question de faire l'analyse de sa pertinence, ni de discuter si cette menace réciproque fût prise au sérieux par les principaux protagonistes, en l'occurrence «Kennedy, McMillan et Khrouchtchev " ${ }^{30}$. Khrouchtchev qui déclarait alors: "Si les États-Unis veulent la guerre, alors nous nous retrouverons en enfer ». Il suffit de savoir, selon Allison ${ }^{31}$ dans sa critique de la Théorie des jeux de von Neumann ${ }^{32}$, que «cette stratégie d'équilibre entre les deux parties ne garantit pas toute attaque nucléaire » et que "The United States might not be in mortal danger but the administration most certainly was" ${ }^{\prime 33}$. Dans sa monumentale Histoire de la Guerre froide Soutou émet les mêmes réserves ${ }^{34}$. Que la menace nucléaire fût ou non bien réelle au niveau des instances de décision, qu'elle le soit encore ou non, n'a pas impacté l'angoisse qui en résultait et qui s'est durablement installée dans les consciences, via le cinéma et les médias. En 1958, l'historien Robert Mandrou soulignait déjà avec force « la place prise par le cinéma dans notre civilisation » et plus loin, il engageait les historiens «à la nécessité de l'étude de l'influence du cinéma sur les comportements collectifs et de prendre la mesure d'une véritable transformation de la sensibilité : les mentalités des hommes du XXe siècle sont directement -et de plus en plus- filles du cinéma, de ses mirages et de ses réalismes ${ }^{35}$. Le cinéma est, dans mon étude, le premier héraut d'une histoire du troisième type où le récit fictionnel se sert immédiatement des événements comme matière à fiction. Il le fait avec ses outils, quitte à prendre des libertés avec le récit historique ${ }^{36}$. Cette frayeur qui, dans ces premières années ressemblait plutôt à une grande peur rationnelle, a fini par s'intérioriser, en grande partie par la puissance du cinéma, telle qu'elle est aujourd'hui présente, dans les médias, avec l'Iran ou la Corée du nord. La menace et l'angoisse d'une guerre nucléaire plane encore.

\footnotetext{
${ }^{29}$ Horne, A., La crise des missiles cubains. In : Histoire, économie et société, 1994, 13e année, $\mathrm{n}^{\circ} 1$. La France et la GrandeBretagne devant les crises internationales. pp. 171-184.DOI : https://doi.org/10.3406/hes.1994.1739 www.persee.fr/doc/hes_07525702_1994_num_13_1_1739. C'est cette journée qui est au cœur de Fail Safe de S. Lumet et de Dr. Strangelove de S. Kubrick.
}

${ }^{30}$ Horne, A., op.cit., 1994.

${ }^{31}$ Allison, G.T., Essence of decision, explaining the Cuban missile Crisis, Harper Collins Publishers, 1971.

${ }^{32}$ Von Neumann à qui est attribué le concept de Mutual Assured Destruction.

33 "Les États-Unis ne sont peut-être pas en danger de mort, mais l'administration l'était certainement". Ma traduction.

${ }^{34}$ D’après Soutou, les soviétiques ne se sont jamais préoccupés de contenir l'équilibre. Les opposants américains au traité SALT2, étaient sûrs «que les Russes recherchaient la supériorité dans une perspective de victoire à l'issue d'une guerre nucléaire ». op.cit., 2001, Chap. XVI pp. 841-844.

\footnotetext{
${ }^{35}$ Mandrou, R., Histoire et Cinéma. In: Annales. Economies, sociétés, civilisations. 13e année, N. 1, 1958. pp. 140-149; doi : https://doi.org/10.3406/ahess.1958.2720.

${ }^{36}$ Delage, C., Guigueno, V., L'Historien et le film, édition Gallimard, 2004. Delage dans le chapitre Pratiques historiennes du cinéma cite Duby : «Je proclame le droit qu'a l'historien d'imaginer. Cependant son devoir est aussi de contenir son rêve dans les limites du connaissable, de demeurer véridique et de veiller à s'interdire tout anachronisme. Ce qui n'est pas une obligation pour le cinéaste. »
} 
Après Yalta, la division du monde en deux blocs hétérogènes que tout opposait et les deux bombes atomiques lâchées sur le Japon par les Américains, à la sortie de la Seconde Guerre mondiale, ont provoqué l'effroi et ont rendu la menace atomique crédible. Le conflit politicoidéologique s'appuyant sur un arsenal nucléaire de plus en plus important, des deux côtés, a provoqué des va-et-vient entre accords de principe et désaccords de fond sur la base d'une limitation de l'armement atomique ${ }^{37}$. Cette situation instable rendait la tension encore plus perceptible et exacerbait les peurs dès le moindre incident. Deux exemples : Le U2-Incident (1960), qui voit cet avion espion américain, abattu par les Russes pour violation du Point limite (Fail Safe) en survolant le territoire soviétique, ou la Crise des missiles de Cuba (1962) qui fut un moment de tension extrême y compris chez les acteurs eux-mêmes (Horne, 1994) ${ }^{38}$ et dont Dr. Strangelove et Fail Safe sont directement et simultanément inspirés. Ces deux incidents firent prendre conscience aux deux Etats de l'urgence d'une période de Détente. Les James Bond cités sont deux bons exemples de ce rapport direct entre une situation politico-militaire qui se veut plutôt relâchée et son illustration cinématographique, puisque l'on y voit un tiers méchant traqué par l'alliance de deux espions que tout devrait opposer. Au contraire, l'intervention soviétique en Afghanistan (1979) qui vient en même temps que le deuxième choc pétrolier $(1979)^{39}$ provoque une tension supplémentaire et intensifie la Seconde Guerre froide. « The Day After (1984) s'en fait l'écho » (Puiseux, 2016).

\section{La menace atomique fait son cinéma}

Le cinéma américain n'a pas attendu la Crise des missiles de Cuba pour produire des films autour de la menace nucléaire. Avant même Hiroshima se produisent et se réalisent toute une série de films de science-fiction sur les risques de radiation. Mais l'approche et la réception publiques changent radicalement après les deux explosions atomiques nipponnes qui révèlent, au monde effrayé, l'usage militaire de l'atome. La crainte alors diffuse de sa domestication à des fins pacifiques devient une angoisse bien réelle dès lors qu'il peut détruire. Au plus fort de la Coexistence pacifique, qui n'a de coexistence et de pacifique que le nom, Stanley Kramer produit et réalise On the Beach (1959) ${ }^{40}$. Le film fait directement référence à la fois à l'usage militaire du nucléaire et à une Troisième Guerre mondiale. A l'autre bout, alors que la tension est remontée entre les deux Etats, The Day After (1983) de Nicholas Meyer reprend jusqu'à son terme irréversible le thème de la destruction au plus fort de la Seconde Guerre froide. Pour bien mesurer les relations de ce récit fictionnel avec l'événementiel, il est nécessaire de dire quelques mots sur celui-ci. Après l'accalmie des relations, c'est, à partir de 1975, le début de très fortes tensions entre l'Est et l'Ouest qui ne vont aller qu'en s'aggravant jusqu'à la rupture. Le risque majeur d'un conflit nucléaire pointe donc à nouveau. Cette période est marquée par un affaiblissement des Etats-Unis, provoqué par l'affaire du Watergate, la chute de Saïgon, la crise économique due au choc pétrolier, face à un accroissement des moyens militaires à l'Est. Les accords SALT2 (1979) ne seront jamais entérinés par le Sénat américain pour cause, entre autres, d'invasion de l'Afghanistan par les troupes soviétiques. Pour les historiens, cette intervention militaire marque à la fois le début de la Seconde Guerre froide en même temps que la fin du conflit entre les deux Etats. C'est, avec l'élection de Ronald Reagan (1980), qui taxe l'Union

\footnotetext{
${ }^{37}$ Installation du « Téléphone rouge » entre Moscou et Washington (1963). Traité sur la non-prolifération des armes nucléaires (1968) et les accords SALT I (1969) et SALT II (1979).

${ }^{38}$ Horne, A., La crise des missiles cubains, in: Histoire, économie et société, 1994, 13e année, $\mathrm{n}^{\circ} 1$. La France et la GrandeBretagne devant les crises internationales. pp. 171-184; doi : https://doi.org/10.3406/hes.1994.1739 https://www.persee.fr/doc/hes_0752-5702_1994_num_13_1_1739
}

\footnotetext{
${ }^{39}$ Les conséquences du premier choc pétrolier (1973) sont au cœur du scénario de The Man with the Golden Gun (1974), le $9^{\mathrm{e}}$ opus de la franchise Bond.
}

${ }^{40}$ Le film est adapté du roman éponyme, de Nevil Shute publié en 1957 
soviétique d'Empire du mal (evil Empire) le renoncement à la Détente, une politique d'augmentation des dépenses militaires, la mise en place de l'IDS (la Guerre des étoiles).

Dans la foulée de l'affaire de l'avion espion et de la Crise d'octobre 1962, paraissent plusieurs films qui s'en inspirent directement. Nous n'avons retenu que les deux premiers et les plus célèbres : Dr. Strangelove: How I Learned to Stop Worrying and Love the Bomb (1964) de Stanley Kubrick et Fail Safe (1964) de Sidney Lumet déclinent le même scénario avec la même fin apocalyptique. Cette crise qui s'est heureusement bien dénouée a provoqué dans l'administration des deux pays une prise de conscience allant jusqu'à engager des négociations pour la non-prolifération des armes nucléaires ; situation qui fait entrer le conflit dans sa période dite de Détente.

Bien que l'angoisse d'une apocalypse nucléaire reste au cœur des deux films suivants, le réchauffement diplomatique entre les Etats-Unis et l'URSS devient sensible dans le choix des synopsis, des personnages et des mises en scène. You Only Live Twice (1967) et The Spy Who Loved $M e(1977)^{41}$ de Lewis Gilbert sortent l'un au meilleur moment de La Détente, l'autre sur son déclin. Le premier, unit les deux Etats contre Spectre qui s'empare des vaisseaux spatiaux russes et américains au profit de la Chine à un moment où le divorce est consommé entre Pékin et Moscou depuis 1965. Il sort après la signature du «Traité d'interdiction partielle des essais nucléaires » (1963) et l'année du Traité de l'espace. L'autre vient après la signature des accords SALT1 (1972) ${ }^{42}$, tout juste après la fin de la Guerre du Vietnam (1975) et après la mission Apollo-Soyouz (1975) qui unit américains et soviétiques dans l'espace, dans une poignée de main devenue célèbre.

Nous voilà donc en présence d'autant de films qui mettent la terreur provoquée par l'apocalypse atomique au cœur même de leurs réalisations. Certains mettent en scène des vies familiales et des romances perturbées, voire anéanties par le conflit jusqu'au mélodrame (On the Beach, The Day After) quand d'autres se limitent à traiter des prises de décisions politiques inédites (Dr. Strangelove et Fail Safe). D'autres jouent sur l'outrance et la désinvolture, mais tout en restant très politique, par allusions iconiques ou textuelles (The Spy Who Loved Me et You Only Live Twice).

\section{On the Beach de Stanley Kramer et The Day After de Nicholas Meyer.}

Ces deux films sont produits et sortent au cours de deux périodes certes distinctes mais proches en termes de tension entre les belligérants -Coexistence pacifique pour l'un et Seconde Guerre froide pour l'autre-. L'un et l'autre exploitent la peur à partir de tensions politiques bien réelles, en s'appuyant sur le souvenir alors bien intégré dans la mémoire collective des images d'horreur d'Hiroshima et Nagasaki. Dans l'un comme dans l'autre la catastrophe a bien lieu. Stanley Kramer situe On the Beach à Melbourne. Des hommes et des femmes attendent la mort dans l'arrivée d'une inéluctable radiation massive provoquée par un cataclysme atomique qui a eu lieu dans l'hémisphère nord. Le réalisateur laisse progressivement l'angoisse des personnages s'installer jusqu'au suicide de masse, par petites touches discrètes sous la forme d'allusions dans le dialogue. Ce sont surtout les mots, des images grises, parfois très sombres et des sons mécaniques qui, par leur répétition, rendent l'atmosphère inquiétante et oppressante. Le film avance par situations domestiques et amoureuses d'une grande banalité dans une lumière sans éclat. Les radiations, invisibles par définition, ne sont présentes que par le son d'un instrument de mesure et dans les propos des personnages. La menace

\footnotetext{
${ }^{41}$ Bien d'autres films anglo-saxons évoquant ce thème sont évidemment sortis au cours de cette période (1963-1979), comme le Torn Curtain (1966) d'Alfred Hitchcock, mais qui ne rend compte que de l'affrontement idéologique et politique entre les deux Etats. D'autres sont plus métaphoriques comme Kiss Me Deadly (1955) de Robert Aldrich ou The Birds (1963) d'Hitchcock. Ces deux films sont, entre autres, cités dans le mémoire remarquable de Thomas Lequeu, «L'angoisse atomique au cinéma. Art et histoire de l'art». 2014. HAL Id:https://dumas.ccsd.cnrs.fr/dumas-01545721. Mais la plupart des productions sont postapocalyptiques comme The Omega Man (1971), A Boy and His Dog (1975) ou Logan's Run (1976), et ne mettent pas en scène le conflit américano-soviétique.
}

${ }^{42}$ Strategic Arms Limitation Talks. SALT1 sont signés le 26 mai 1972. SALT2, le 18 juin 1979. 
est là, bien réelle, mais invisible. C'est cette invisibilité et l'inéluctabilité de la mort prochaine qui crée toute la tension et produit chez les personnages et chez le spectateur une sensation d'oppression. Afin d'éviter une mort certaine, lente et douloureuse, le gouvernement australien distribue massivement des pilules dont l'absorption assure une mort rapide. Ce qui a pour effet de mettre le spectateur dans une position d'identification avec les personnages. Que ferais-je dans cette situation? Est-ce que je choisirais le suicide proposé ou bien est-ce que j'attendrais cette mort inconnue par radiation? Le film se termine sur des images grises, sans contrastes, des rues désertes et désolées de San-Francisco vues à l'aide du périscope par les rares survivants d'un sous-marin américain. Au final, ce qui rend l'atmosphère du film la plus oppressante, c'est son parti pris esthétique d'images sans relief, presque documentaires. Il importe de noter une sorte de prémonition des films à venir, puisqu'un plan, plein cadre montre, au tout début de la narration, le calendrier de l'année 1964, date de sortie du Kubrick et du Lumet. Ce détail révèle ce temps suspendu que décrit le film mais aussi l'acuité du regard de Kramer sur les affaires du monde, son intérêt pour la chose politique et une saisie de ce qui « dans l'air » peut conduire à l'inévitable.

The Day After est produit et diffusé par la télévision américaine en 1983 et en France en 1984. Dans le Meyer, l'URSS envahit l'Europe de l'Ouest déclenchant ainsi une inévitable guerre nucléaire. The Day After est exemplaire du film de propagande et des liens forts entre le récit fictionnel et la situation politique. Le film en couleurs sans éclat, déploie tous les clichés de l'American Way of Life avant la catastrophe. Les premières minutes nous montrent un peuple heureux dans des paysages ruraux ou urbains paradisiaques : des agriculteurs, des cow-boys, des écoliers, un jeune couple prêt à se marier, des parents heureux, jusqu'au match de football entre jeunes gens avec deux pom-pom girls en bord de touche. Toutes ces images sont accompagnées de ces airs musicaux qui courent dans les westerns et les comédies romantiques américaines. Home sweet home, «Vive l'Amérique » crie le film. Mais « L'Empire du mal » provoque. Il envahit non pas l'Afghanistan, sans doute trop loin culturellement, mais l'alliée, l'Europe de l'Ouest et il s'apprête à attaquer les Etats-Unis. Les Soviétiques sont nommément accusés de vouloir détruire cette Nation enjouée, prospère et démocratique. L'inquiétude grandit peu à peu, elle s'installe de façon larvée venant semer le trouble dans ces vies si heureuses et si tranquilles. Le film change alors de tonalité, passant des visages sereins et souriants aux regards angoissés. Les images et les dialogues nous font revenir trente années en arrière au plus fortes et aux plus caricaturales images de la Coexistence pacifique. Le suspense est maintenu par les diverses tentatives pathétiques d'hommes, de femmes et d'enfants, pour échapper à la mort. Le film s'achève dans les mêmes paysages ruraux et urbains du début, mais en ruine, dévastés par les explosions atomiques, les champs et les rues parsemés de cadavres. Afin de bien marquer la cruauté de l'ennemi diabolique, Meyer s'attache à suivre ce qui reste d'enfants, de femmes et d'hommes américains, dans leurs efforts de survie. On the Beach et The Day After se passent l'un et l'autre en extérieur et découvrent un monde dévasté après les explosions qui, en anticipant, vont clôturer les deux films suivants.

\section{Dr. Strangelove, de Stanley Kubrick et Fail Safe de Sidney Lumet.}

Kubrick et Lumet s'emparent et transforment un événement historique ${ }^{43}$ qui aurait pu se terminer par une catastrophe. Les deux scripts mettent en scène l'angoisse du président devant la Nuclear $F_{o o t b a l l}{ }^{4}$. Un général américain a décidé de lancer une attaque nucléaire sur l'URSS et de part et d'autre du Rideau de fer, c'est la tentative désespérée d'y mettre un terme. Outre la renommée de

\footnotetext{
${ }^{43}$ Le délai entre l'affaire des missiles et la sortie des deux films est trop court pour que ce ne soit que l'affaire des missiles de Cuba qui ait pu donner aux deux cinéastes l'idée de faire ces films. Il est fort probable qu'il s'agisse plutôt des deux affaires l'avion espion et les missiles de Cuba- décalées entre la mise en écriture, la production et la sortie des deux films.

${ }^{44}$ Nuclear football ou tout simplement football est la mallette contenant les codes nucléaires. Aux Etats-Unis elle suit le président dans tous ses déplacements. Daniel F. Ford, The Button: The Pentagon's Strategic Command and Control System, Simon and Schuster, 1985.
} 
ces deux films et de leurs auteurs, ce choix se justifie par la proximité des scénarios, par la société Columbia qui, au final, produit ces deux films la même année et par la différence de traitement cinématographique. Si les deux œuvres mettent pareillement en scène la prise de décision présidentielle dans une situation inédite et tragique, le film de Kubrick est chargé d'ironie, quand celui de Lumet est funeste.

Le Kubrick alterne des séquences du centre de contrôle où siège, autour du président, un staff de conseillers militaires. Coupé de ce centre de contrôle, le dernier bombardier continue sa mission jusqu'à larguer ses bombes dans une symphonie de champignons nucléaires. Comme le sous-titre l'indique, le film s'emploie à dénoncer avec une cruelle ironie et un humour ravageur, jusqu'à la caricature, dont l'acronyme MAD se transforme dans le film en mad (fou), l'impéritie des politiques et des militaires à prendre quelques décisions que ce soient, ou alors de bien mauvaises et dangereuses. Le script met en scène un président des Etats-Unis bedonnant, à qui Kubrick donne les traits du physicien John von Neumann. Il est entouré de trois conseillers : un colonel butor et borné qui a la charge de le conseiller militairement, et afin de mieux souligner ce qui pour Kubrick relève d'une irresponsabilité partagée, incarné par un seul et même acteur -Peter Sellers- un militaire britannique et un scientifique cloué sur un fauteuil roulant, présentant tous les traits d'un savant nazi qui renvoie à l'Opération Paperclip ${ }^{45}$. L'angoisse qui n'est ressentie que par les membres du Conseil de Guerre siégeant dans la vaste Salle des opérations, contraste avec le laconisme du duo Clown-blanc-président et son Auguste-colonel-conseiller. La tension reste plus moquée que réelle, cantonnée à l'intérieur de l'action d'une œuvre satirique. Chez Lumet la gravité de la situation et la responsabilité du président des Etats-Unis de négocier avec son homologue soviétique est mise en scène de manière dramatique, quand Kubrick les fait dialoguer sur le ton de la comédie de boulevard. Au contraire du président kubrickien, celui de Lumet est incarné par Henri Fonda, acteur populaire au physique avenant, enfermé une grande partie du film, au secret, avec un seul conseiller grave et paniqué. Cependant, outre le script, il existe de nombreux points communs entre les deux films. Les deux réalisateurs ont fait le choix d'un quasi huis clos et d'une image très contrastée, en jouant sur toutes les possibilités plastiques et oppressantes du noir et blanc, ainsi que celui de structurer leur récit en montage alterné entre le centre de décision et les bombardiers dans leur mission de destruction et de mettre en scène les négociations entre présidents. Ces mêmes parti pris ne produisent pourtant pas les mêmes effets. Quand le générique de Dr. Strangelove s'incruste dans les airs avec deux bombardiers survolant une mer de nuages sur une musique douce, celui de Fail safe met en scène un cauchemar, une corrida avec mise à mort du taureau, filmée au plus près du corps de l'animal. C'est sur ces deux modes que les films progressent. La tension est nette et palpable chez les deux principaux protagonistes de Fail Safe en un huis-clos rendu encore plus étouffant par l'exiguïté du lieu et des gros plans sur des visages blêmes et transpirants. Là où Kubrick va accompagner son film par un leitmotiv musical du folklore américain, Lumet ne joue que sur les sons de machines, d'avions, de sonnerie de téléphones sans ajout musical. L'usage des contre-plongées, nombreuses dans les deux films, contribue à créer une sensation d'oppression chez Lumet et de grotesque chez Kubrick. Le final, identique dans le script, est traité très différemment par l'un et l'autre. Le dernier plan du film sur lequel va se dérouler, en silence, le générique de Fail Safe est une image noire au centre de laquelle se devine une très vague forme blanche et floue qui pourrait être celle du champignon atomique. Tandis que Kubrick, après avoir introduit l'image archétypale du cow-boy chevauchant la bombe, crée une sarabande d'explosions sur la chanson "We'll meet again/Don't know where/Don't know when/But I know we'll meet again some sunny day/Keep smiling through/Just like you always do/'Till the blue skies drive the dark clouds far

\footnotetext{
${ }^{45}$ Opération secrète de l'armée américaine qui consistait à exfiltrer des centaines de savants allemands et de les soustraire, au moins pour certains d'entre eux comme le SS Werner von Braun aux procès de Nuremberg. Von Braun qui deviendra l'un des directeurs des programmes spatiaux américains
} 
away ?"46 Si celui-ci convoque l'événement, c'est pour le mettre à distance par dérision, quand celui-là l'utilise pour provoquer plus d'angoisse encore. A la suite des deux incidents évoqués par ces deux œuvres artistiques s'ouvre la période historique de la Détente.

\section{You Only Live Twice et The Spy Who Loved Me (franchise James Bond), de Lewis Gilbert}

Dans la mesure où les aventures de l'agent secret britannique constituent une franchise, je vais d'abord dégager ce que ces deux films ont en commun. Puis je m'attacherai à mettre en évidence ce qui les différencie en regard de la situation internationale. Les multiples films mettant en scène James Bond, ont de nombreux points communs qui vont bien au-delà du seul héros principal. Les récits sont structurés de la même manière. Selon Eco, qui fait une analyse des livres de Fleming que l'on peut transposer aux films malgré quelques libertés prises par les scénaristes : «La trame...est grosso-modo la suivante : Bond est envoyé dans un endroit donné pour éventer un plan de type science-fiction, ourdi par un individu monstrueux d'origine incertaine, en tout cas pas anglais, qui, utilisant une activité propre soit comme producteur soit comme chef d'une organisation, non seulement gagne énormément d'argent, mais fait le jeu des ennemis de l'Occident. En allant affronter cet être monstrueux, Bond rencontre une femme dominée par lui et la libère de son passé en établissant avec elle un rapport érotique, interrompu par la capture de Bond par le Méchant et par la torture qui lui est infligée. Mais Bond défait le Méchant qui meurt de façon horrible, puis il se repose de ses dures fatigues entre les bras de la femme, qu'il est toutefois destiné à perdre ». Une fois retirées toutes les « séquences-poncifs» (Nardone, 2020) ${ }^{47}$ ou les «situations de jeu » (Eco, $1966)^{48}$ qui constituent environ les trois-quarts des James Bond, apparaissent les tiers méchants, leur implication dans la menace et la mission de l'agent 007 (sauver le monde d'un cataclysme nucléaire). Ici, le suspense est réduit à sa plus simple expression, dans la mesure où le spectateur détendu en connait l'issue. «La trame véritable demeure immuable et le « suspense » s'établit de façon curieuse sur une suite d'événements entièrement escomptés » $(\text { Eco, 1966 })^{49}$. Eco a raison de dire que le spectateur connait bien évidemment l'issue de la mission, mais il reste suspendu aux péripéties qui vont conduire le héros à la réussir. Les deux films de Gilbert, ont en commun de s'inspirer du rapprochement entre les deux superpuissances, pour raconter une histoire d'espionnage qui mêle relation amoureuse et menace nucléaire. Les tensions n'y sont que diluées, la caricature prend le pas sur le sérieux de la menace pourtant présente. Nous sommes bien dans cette période historique dite de la Détente où le risque nucléaire s'est alors déplacé du réel vers le seul fictionnel. Il subsiste encore, mais dans la fable divertissante. La bombe est là, le danger est bien là, mais ils sont relégués au second plan. Ces deux films sont pour le spectateur des grands moments de divertissement. Dérogeant à mon engagement de ne m'en tenir qu'au seul objet film, je tiens à dire quelques mots sur les conditions de production de ces deux épisodes de la série. Ils sont, comme tous les Bond, produits par la société EON Production. Lors de la mise en production de You Only Live Twice (1967), EON est encore essentiellement britannique, même si les films sont diffusés par la société américaine United Artists qui gère les droits de la franchise. C'est donc à cette époque, où la menace d'une guerre nucléaire est momentanément écartée, que le film est produit. Mais le monde est loin d'être apaisé. Entre 1964 et 1968, la guerre du Vietnam s'intensifie. Des armes et des munitions venant d'Union soviétique transitent par la Chine vers le Vietnam. L'URSS et la Chine ont rompu officiellement leurs relations en 1961, et des tensions frontalières graves se poursuivent

\footnotetext{
${ }^{46}$ Nous nous reverrons/Je ne sais pas où/Je ne sais pas quand/Mais je sais que nous nous reverrons un jour ensoleillé/Continuez à sourire/Comme vous le faites toujours/Jusqu'à ce que le ciel bleu éloigne les nuages sombres. Notre traduction.

${ }^{47}$ Nardone, R., Le récit de la "Conquête spatiale » op.cit. J'entends par là, comme Eco pour les romans, ces séquences transposables d'un film à l'autre -poursuites diverses, mises en danger de Bond, romance, Casino, Miss Moneypenny, gadgets, etc.- qui sont le propre des séries.

${ }^{48}$ Eco, U., op.cit., 1966.

${ }^{49}$ Eco, U., op.cit., 1966, p.15.
} 
entre ces deux pays, jusqu'à la mort de Mao en 1976. Que nous dit le script de You Only Live Twice?

James Bond doit trouver et arrêter le coupable d'une série de détournements spatiaux aussi bien russes qu'américains. Le Spectre dont l'identité est peu définie met donc sa puissance technique digne d'un Etat, au service de la République populaire de Chine dont l'objectif est de déclencher une guerre nucléaire qui détruira les Etats-Unis et l'URSS. « Ainsi, la Russie cesse d'être l'ennemi habituel, du fait de la détente internationale, et le rôle d'organisation maléfique est repris par le Spectre. Le Spectre a toutefois toutes les caractéristiques du SMERSH ${ }^{50}$, y compris l'emploi d'éléments slavo-latino-allemands, les méthodes de torture et d'intimidation, la haine jurée aux Puissances du Monde libre » (Eco, 1966). Du personnage le spectateur ne peut voir que des mains baguées caressant un chat blanc aux yeux verts, jusqu'à ce que Bond le démasque. Il apparait alors le visage barré d'une longue cicatrice. Les soldats de son armée portent des uniformes rouges ou jaunes, reprenant ainsi les couleurs des drapeaux chinois et soviétique. Le stratagème employé pour entrainer les deux superpuissances à s'anéantir, consiste à enlever les vaisseaux spatiaux habités de l'un et de l'autre, au moyen d'un immense engin volant qui les avale littéralement. Nous sommes alors en pleine compétition spatiale entre les deux nations. Il ne fait donc aucun doute pour l'un que c'est l'autre qui est responsable de ces disparitions. Les services secrets britanniques soupçonnant l'implication japonaise, Bond se rend au Japon où il obtient l'aide des services secrets japonais en la personne de leur chef et de son assistante la belle James Bond girl Aki. Après toute une série de séquences, les plus rocambolesques les unes que les autres, Bond aidé d'Aki sauvera le monde d'une catastrophe nucléaire en détruisant le repaire volcanique du Méchant.

\section{The Spy Who Loved Me (1977)}

Entre la production précédente et celle-ci, EON Productions est reprise par la société américaine United Artists (U.A) en 1975, qui détenait déjà les droits de la franchise. Bien qu'elle semble échapper à ma problématique, cette information n'est pas anodine dans la décision de faire ce filmlà précisément et avec le même réalisateur. L'arrivée sur le marché du film de tentpole pictures, films d'action à gros budget, comme les Dents de la mer ${ }^{51}$ (1975) où le suspense et la terreur se sont déplacés du nucléaire et de la Guerre froide vers une menace plus métaphorique détrônent les Bond en nombre d'entrées jusqu'à mettre EON Productions en difficultés financières avant la reprise par U.A. et la mise en chantier de The Spy Who Loved Me (1977). Le script est un remake du précédent qui fut, dix ans plus tôt, l'un des succès commerciaux de la franchise. Il unit, par la volonté de leurs chefs respectifs, l'agent britannique James Bond et l'agent soviétique Anya Amasova. Le prégénérique montre Bond qui tente d'échapper à un commando du KGB tout de noir vêtu au cours d'une délirante poursuite à skis dans un décor alpin somptueux et virginal. A l'issue de cette séquence Bond tue le fiancé d'Anya qui commandait le groupe de tueurs du KGB. Ce pré-générique qui vient donc avant la mission proprement dite présente un double intérêt pour la suite. Il montre bien l'état de tension entre les deux superpuissances tel qu'il l'était avant ce que nous allons voir. Par la mort du fiancé d'Anya, il nous indique que l'association des deux agents est contre nature et qu'elle ne va pas se faire spontanément. Ce qui permet, l'air de rien, de faire passer le message : nous sommes bien dans une période de détente entre nos deux nations pour une mission commune mais nous restons deux camps ennemis. Ladite mission consiste à retrouver des sous-marins nucléaires russes et britanniques qui ont mystérieusement disparu. Le milliardaire Karl Stromberg, le méchant du film, compte se servir des sous-marins nucléaires qu'il a volés pour détruire le monde

\footnotetext{
${ }^{50}$ SMERSH, « mort aux espions » était le nom utilisé pour désigner les départements de contre-espionnage de l'armée soviétique durant la Seconde Guerre mondiale.

${ }^{51}$ Le film rapporta 470 millions de dollars dans le monde et fut pendant deux ans le plus gros succès du cinéma américain. Il sera suivi deux ans plus tard par Star Wars. Ces films qui apparaissent en pleine Détente, inaugurent de façon plus métaphorique encore que ne le font les Bond, au cours de cette même période, en déplaçant le terrain du conflit entre nations.
} 
et créer une cité sous-marine baptisée Atlantis. Le Méchant menace à nouveau la planète d'un enfer atomique grâce à un plan qui ressemble trait pour trait à celui imaginé par le Spectre de You Only Live Twice. Le script remplace le super vaisseau spatial, engloutissant les vaisseaux spatiaux russes et américains, par un supertanker, engloutissant les vaisseaux sous-marins russes et britanniques. Il substitue la conquête spatiale par la conquête des océans et le bassin aux piranhas par le bassin aux requins. Là où il n'était donné à voir au spectateur du Méchant, qu'une main baguée caressant un chat aux yeux verts, il découvre ici le visage impassible de l'acteur allemand Curd Jurgens, au regard bleu glacial. Là où les costumes de l'armée de Spectre étaient jaunes et rouges, ceux du méchant Stromberg ${ }^{52}$ sont entièrement rouges. Au final Bond sauvera le monde Libre, après de mêmes aventures tout aussi invraisemblables, dans la même explosion détruisant les installations meurtrières du Méchant.

Ce film vient répondre doublement à ma problématique. Il y répond d'abord par son script qui associe (jusqu'à la romance) un agent du monde dit Libre avec un agent de l'Empire du mal pour une même mission. Ce scénario est tout à fait impensable en dehors de cette période. Mais ce qui le différencie du précédent tient à la situation mondiale qui voit se profiler depuis les deux dernières années, de nouvelles tensions entre les deux superpuissances, menaçant la Détente. Nous sommes en 1977 et comme je l'ai déjà noté, l'Occident est entré dans une ère de récession économique avec la crise pétrolière (1973). Avec la fin de la guerre du Vietnam (1975), qui constitue un échec des EtatsUnis, et après l'affaire du Watergate (1974), qui jette l'opprobre sur l'administration américaine, le Congrès adopte une politique plus isolationniste et plus dure avec l'URSS. A l'Est, Brejnev abandonne la politique de Détente. L'heure est alors au doute et à la peur.

\section{Conclusion}

Le film de guerre est un genre en soi, mais peu d'entre eux ont ce pouvoir d'interférer à ce point avec une réalité qui concerne l'ensemble de l'humanité en train de la vivre dans l'angoisse. Hélène Puiseux nous dit qu'ici « Une conception complètement différente dans la réalisation et les scénarios apparaît avec les récits sur l'arme nucléaire [...] Ce discours pallie la perspective insupportable d'une catastrophe finale inévitable [...] en habituant le spectateur à admettre l'arme atomique dans son espace de pensée. On rend l'atome racontable, donc admissible, on lui donne une histoire imaginaire qui se déroule pendant qu'il en acquiert une réelle sur terre, avec les essais et la Guerre froide, qui menaçait à toute occasion de tourner en apocalypse nucléaire réelle. ${ }^{53} \mathrm{Ce}$ qui me conduit une nouvelle fois à examiner ces productions artistiques comme potentiel vecteur de propagande à chacun des moments précis des relations. "La propagande est utile au politicien si, et seulement si, le public, consciemment ou non, a envie d'entendre ce qu'il a à dire. ${ }^{54}$ Encore faut-il qu'il soit préparé. Les films de cinéma n'agissent jamais seuls, ils ne s'inscrivent pas seulement dans un contexte historique mais aussi dans un imaginaire collectif structuré par des institutions qui est prêt à les recevoir (Odin, 2000 ; Gell, 1998) ${ }^{55}$. C'est bien toute la problématique que j'ai fait défiler qui est présente dans mon corpus. Ce qui paraît remarquable dans le contexte de la Guerre froide et de la menace nucléaire, c'est que ce cinéma-là comme le souligne Puiseux, construit cet imaginaire, en même temps qu'il découvre la portée symbolique de ce conflit politico-militaire singulier, associé à une arme inédite dont la menace radioactive est invisible mais capable de

\footnotetext{
${ }^{52}$ Pour l'analyse des noms des méchants dans la série des Bond, on lira avec intérêt l'article cité d'Umberto Eco.

${ }^{53}$ Puiseux, H., Problèmes d'analyse cinématographique : La perspective mythologique In: École pratique des hautes études, Section des sciences religieuses. Annuaire. Tome 111, 2002-2003. 2002. pp. 31-56; doi https://doi.org/10.3406/ephe.2002.12043https://www.persee.fr/doc/ephe_0000-0002_2002_num_115_111_12043

${ }^{54}$ Bernays, E., Propaganda, La Découverte, 2007, p.160.

${ }^{55}$ Odin, R, De la fiction, op.cit., 2000.
} 
provoquer le néant. Outre que l'analyse des films montre bien l'affinité des scénarios avec les moments du conflit, elle révèle, comme j'en faisais l'hypothèse, la diversité des discours dans une même période. Il est difficile de voir On the Beach comme un film de propagande pro-américain, même s'il se termine sur des images de San Francisco en ruine. Il en est de même pour l'ironique Dr. Strangelove ainsi que pour le très grave Fail Safe, quand The Day After présente toutes les caractéristiques d'un film de propagande américain anticommuniste. De l'analyse qu'Umberto Eco fait des méchants dans les Bond, se dégage une possible tentation d'y voir le même type de propagande qui passerait, entre autres, par les noms et les origines des personnages les incarnant. Il nous dit plus loin «qu'il est difficile, après l'analyse que nous avons menée, de nier que Fleming incline à penser que l'homme anglo-saxon est supérieur aux races orientales ou méditerranéennes, ou qu'il professe un anticommunisme viscéral. Il est toutefois remarquable qu'il cesse d'identifier le mal avec la Russie dès que la situation internationale permet de moins la craindre selon la conscience du commun ». Cette dernière remarque d'Eco marque bien ce que j'ai mis en évidence dans les deux films bondiens. Ce qui confirme l'hypothèse de pouvoir déceler l'état des relations entre les deux superpuissances pendant la Guerre froide à l'aune des films mettant en scène une possible eschatologie nucléaire. Il semble même pertinent d'ajouter, comme j'en faisais l'hypothèse, que ces films sont de véritables représentations indirectes d'une série de conditions géopolitiques et géoculturelles, avant d'être des outils de propagande en soi.

A la fin de la Guerre froide, l'eschatologie nucléaire n'en n'a pas moins été au centre de quelques films hollywoodiens. Sur la fin du conflit, on trouve des films qui évoquent des nostalgiques de cette période comme The Peacemaker (1997) ou encore The Sum of All Fears (2002). Puis elle se déplace, comme je le notais plus haut, vers d'autres ennemis potentiels ou métaphoriques. La menace vient alors de l'espace Deep Impact (1998) ; mais elle peut venir aussi bien des machines, comme en témoigne Outside the Wire (2021), Stealth (2005) et Terminator 3 (2003), ou bien du terrorisme comme dans Next (2007) ; ou bien l'apocalypse a déjà eu lieu comme dans Equilibrium (2002) dont le titre fait bien référence à l'équilibre de la terreur de la Guerre froide.

\section{Bibliographie indicative}

Allison G.T., Essence of decision explaining the Cuban missile Crisis, Harper Collins Publishers, 1971.

De Baecque A., L'Histoire-caméra, Gallimard, Paris, 2008.

Baroni, R., «Le cliffhanger : un révélateur des fonctions du récit mimétique », Cahiers de Narratologie [En ligne], 31 | 2016, mis en ligne le 22 décembre 2016, consulté le 20 février 2020. URL: http://journals.openedition.org/narratologie/7570 ; DOI : https://doi.org/10.4000/narratologie.7570

Barron, E.N., Game Theory, an Introduction, Wiley-Blackwell, 2013.

Bazin A., Qu'est-ce que le cinéma ? Editions du Cerf, Paris, 2010.

Bessalel, J., Gardies, A., (dir.), Le suspense au cinéma, CinémAction, n 71, Corlet Publications, 1994,

Bellour R., L'Analyse du film, Calmann-Lévy, Paris, 1995.

Bellour R., Le corps du cinéma. Hypnoses, émotions, animalités, POL éditeur, Paris, 2009.

Cayatte, R., et Goudmand, A., «Approches transmédiales du récit dans les fictions contemporaines », Cahiers de Narratologie [En ligne], $37 \quad 2020$, mis en ligne le 04 septembre 2020, URL : http://journals.openedition.org/narratologie/10392 ; DOI : https://doi.org/10.4000/narratologie.10392

Crozier M., E. Friedberg, L'acteur et le système, Editions du Seuil, Paris, 1977.

De Baecque, A. L'Histoire-caméra, Gallimard, Paris, 2008.

Delage C. \& V. Guigueno, L'historien et le film, Gallimard, Paris, 2004.

Dewey J., Le public et ses problèmes, Editions « Tractacus \& Co », Paris, 2005.

Eco, U., James Bond : une combinatoire narrative. In: Communications, 8, 1966. Recherches sémiologiques : l'analyse structurale du récit. pp. 77-93; doi : https://doi.org/10.3406/comm.1966.1116 https://www.persee.fr/doc/comm_0588-8018_1966_num_8_1_1116 
Encyclopédie de la philosophie, Librairie Générale Française, 2002.

Ferro, M., Cinéma et Histoire, Denoël et Gonthier, Paris, 1977.

Ferro, M., Analyse de film, analyse de sociétés : une source nouvelle pour l'Histoire, Hachette, Paris, 1974.

Ferro, M., Film et histoire, Éditions de l'EHESS, Paris, 1984.

Fohlen, C., Heffer, J., \& Weil, F., (1997). De la diplomatie aux relations internationales. Dans : C. Fohlen, J. Heffer \& F. Weil (Dir), Canada et États-Unis depuis 1770 (pp. 413-448). Paris cedex 14, France: Presses Universitaires de France. Graham T. Allison, Essence of Decision: Explaining the Cuban Missile Crisis, Little Brown, 1971.

Ford D. F., The Button: The Pentagon's Strategic Command and Control System, Simon and Schuster, N.Y, 1985.

Gaddis J.L, The Cold War: A New History, New York, NY, The Penguin Press, 2005.

Gell, A., Art and Agency: An Anthropological Theory, Oxford: Clarendon, 1998.

Histoire, économie et société, 1994, 13e année, n 1 . La France et la Grande-Bretagne devant les crises internationales. $\mathrm{n}^{\circ}$. La France et la Grande-Bretagne devant les crises internationales. pp. 171-184; doi : https://doi.org/10.3406/hes.1994.1739.

Horne A., La crise des missiles cubains. In : Histoire, économie et société, 1994, 13e année.

Lacorne, D., «Une laïcité à l'américaine », Études, 2008/10 (Tome 409), p. 297-305. DOI : 10.3917/etu.094.0297. URL : https://www.cairn.info/revue-etudes-2008-10-page-297.htm.

Lalande A., Vocabulaire technique et critique de la philosophique, Vol I, $5^{\mathrm{e} e ́ d i t i o n . ~ « Q u a d r i g e ~ » ~ P U F, ~ P a r i s, ~} 1999$.

Lepri C., " De l'usage des médias à des fins de propagande pendant la guerre froide », in Revue internationale et stratégique, 2010/2 ( ${ }^{\circ}$ 78), p. 111-118. DOI : 10.3917/ris.078.0111. URL : https://www.cairn.info/revueinternationale-et-strategique-2010-2-page-111.htm.

Les Institutions de l'image, sous la direction de Bertin-Maghit J.P et Fleury-Vilatte B., Editions de l'EHESS, Paris, 2001.

Mandrou, R. Histoire et Cinéma. In: Annales. Economies, sociétés, civilisations. 13e année, N. 1, 1958. pp. 140-149; doi : https://doi.org/10.3406/ahess.1958.2720.

Mario R., Les relations américano-soviétiques. In : Politique étrangère, nº5 - 1976 - 41eannée.

Metz C., Essais sur la signification au cinéma, T.1\&2, Editions Klincksieck, Paris, 1972.

Mitry J., Esthétique et psychologie du cinéma, les Editions du Cerf, 2001.

Natanson, J. (2008). La peur et l'angoisse. Imaginaire \& Inconscient, 22(2), 161-173. doi:10.3917/imin.022.0161.

Nelkin D., La crainte du nucléaire. Éditions CRCT [Centre de Recherche sur la Culture Technique] Cornell University. 1983.

Odin, R, De la fiction, De Boeck Université, Bruxelles, 2000

Parmentier G., Le retour de l'Histoire. Stratégie et relations internationales pendant et après la Guerre froide. Éditions Complexe (programme ReLIRE), in "Questions au XXe siècle », 1993, 306 pages. ISBN : 9782870274835 . DOI : 10.3917/comp.parme.1993.01. URL : https://www.cairn.info/le-retour-de-1-histoire--9782870274835.htm.

Puiseux H., L'apocalypse nucléaire et son cinéma, Cerf, col. 7e Art, Paris, 1988.

Puiseux, H., Problèmes d'analyse cinématographique : La perspective mythologique In: École pratique des hautes études, Section des sciences religieuses. Annuaire. Tome 111, 2002-2003. 2002. pp. 31-56; doi https://doi.org/10.3406/ephe.2002.12043https://www.persee.fr/doc/ephe_0000-0002_2002_num_115_111_12043

Rossellini R., Le cinéma révélé, Editions de l’Etoile, Flammarion, Paris, 1984.

Soutou G-H., La Guerre de Cinquante Ans, Les relations Est-Ouest, 1943-1990, Fayard, Paris, 2001.

Tertrais, B., L'arme nucléaire, Presses Universitaires de France, Paris, 2008.

Truffaut, F., Hitchcock/Truffaut, Ramsay, Paris, 1983.

Truman H.-S., Letter to Senator Flanders on the Appropriation for the Campaign of Truth, 30 août 1950, http://trumanlibrary.org/publicpapers/viewpapers.php?pid=856. 
Vassilis K., L'angoisse. Presses Universitaires de France, « Que sais-je ? », 2017, 128 pages. ISBN : 9782130789314. URL : https://www.cairn.info/l-angoisse--9782130789314.htm

Vernier J.-M., Cinéma et Amérique : une image effritée. In: Quaderni, n50-51, Printemps 2003. Images de l'Amérique $d u$ Nord vues par elle-même ou vues par les autres pp. 197-209. DOI : https://doi.org/10.3406/quad.2003.1228,www.persee.fr/doc/quad_0987-1381_2003_num_50_1_1228

Westad, Odd Arne, The Cold War: A World History, Allen Lane, 31 août 2017, 720 p. (ISBN 978-0241011317).

Traduction en français sous le titre Histoire mondiale de la guerre froide. 1890-1991, Perrin, Paris, 2019. 